\title{
Molecularly imprinted polymers-coated gold nanoclusters for fluorescent detection of bisphenol A
}

\author{
Xiaqing $\mathrm{Wu}^{\mathrm{a}, \mathrm{b}}$, Zhong Zhang ${ }^{\mathrm{b}, \mathrm{e}}$, Jinhua $\mathrm{Li}^{\mathrm{b}}$, Huiyan You ${ }^{\mathrm{a}, *}$, Yanbin $\mathrm{Li}^{\mathrm{c}, \mathrm{d}}$, Lingxin Chen ${ }^{\mathrm{b}, * *}$ \\ anvironmental and Chemical Engineering College, Dalian University, Dalian 116622, China \\ ${ }^{\mathrm{b}}$ Key Laboratory of Coastal Environmental Processes and Ecological Remediation, Yantai Institute of Coastal Zone Research, Chinese Academy of Sciences, \\ Yantai 264003, China \\ c College of Biosystems Engineering and Food Science, Zhejiang University, Hangzhou 310058, China \\ ${ }^{\mathrm{d}}$ Department of Biological and Agricultural Engineering, University of Arkansas, Fayetteville, AR 72701, USA \\ e University of Chinese Academy of Sciences, Beijing 100049, China
}

\section{A R T I C L E I N F O}

\section{Article history:}

Received 7 November 2014

Received in revised form 23 January 2015

Accepted 28 January 2015

Available online 7 February 2015

\section{Keywords:}

Gold nanoclusters

Molecularly imprinted polymers

Fluorescent detection

Bisphenol A

\begin{abstract}
A B S T R A C T
A flexible fluorescent sensing strategy for the recognition and detection of bisphenol A (BPA) has been proposed based on molecularly imprinted polymers (MIPs)-coated gold nanoclusters (AuNCs), by taking advantages of the high selectivity of MIPs and the strong fluorescence property of AuNCs. $\mathrm{SiO}_{2} @ A u N C s$ were initially prepared by making use of the powerful amido bonds between carboxyl-terminated AuNCs and amino-functionalized $\mathrm{SiO}_{2}$ nanoparticles. Then MIPs-coated AuNCs were formed by anchoring MIP layer on the surface of $\mathrm{SiO}_{2} @ A u N C s$ via a sol-gel process. In the presence of imprinting template BPA, a Meisenheimer complex could be formed between BPA and the primary amino groups on the surface of the AuNCs, and the photoluminescent energy of AuNCs would be transferred to the complex, and thereby result in the fluorescence quenching of AuNCs. The fluorescence-quenching fractions of the sensor presented a satisfactory linearity with BPA concentrations over the range of $0-13.1 \mu \mathrm{M}$ and the detection limit could reach $0.10 \mu \mathrm{M}$. Distinguished selectivity was also exhibited to BPA over other possibly competing molecules. Moreover, the sensor was successfully applied to determine BPA in seawater, and the average recoveries of BPA at three spiking levels ranged from 91.3 to $96.2 \%$ with relative standard deviations below 4.8\%. This AuNCs-MIPs based sensor provided great potentials for recognition and determination of phenolic environmental estrogens in complicated samples.
\end{abstract}

(c) 2015 Elsevier B.V. All rights reserved.

\section{Introduction}

Gold nanoclusters (AuNCs) have strong luminescence emission due to the aggregation-induced emission (AIE) with a quantum yield about $15 \%$, and possess remarkable advantages such as small size, mild preparation conditions and non-poisonous properties [1]. Compared with existing widely used fluorescent probes, especially, quantum dots (QDs) such as CdTe [2,3] and CdSe [4], AuNCs exhibit stronger and more durable fluorescence signals attributing to better resistance to photobleaching and blinking [5]. Therefore, AuNCs have attracted even more attentions and gradually become ideal fluorescent sensor candidates [6-8]. For example, a labelfree method for the detection of $\mathrm{Hg}^{2+}$ ions with high selectivity and sensitivity has been developed by using fluorescent AuNCs

\footnotetext{
* Corresponding author. Tel.: +86 411 87402440; fax: +86 41187402436 .

** Corresponding author. Tel.: +86 535 2109130; fax: +86 5352109130.

E-mail addresses: dlyhy@dicp.ac.cn (H. You), lxchen@yic.ac.cn (L. Chen).
}

[7]. Biomolecule-stabilized AuNCs as a fluorescence probe has been reported for sensitive and selective detection of glucose [8]. So, we expect to utilize the outstanding AuNCs material to perform related fluorescent analysis studies.

Meanwhile, another attractive material, molecularly imprinted polymers (MIPs) with high stability, ease of preparation and low cost have flourished in recent decades, particularly displaying promising application potentials in chemo/biosensors [9-11]. Among them, surface MIPs (SMIPs) with core-shell hybrid structure have many advantages over the traditional MIPs, such as more complete removal and more easily rebinding of templates, more accessible recognition sites, and better-defined material morphologies [12]. SMIPs coupled with QDs based sensors have boomed [13-16]. For instance, Liu et al. synthesized Mn-doped ZnS QDs capped by MIPs toward the template molecule 4-nitrophenol detection [14]. Zhou et al. fabricated a fluorescent sensor by anchoring the MIP layer on the silica-coated graphene QDs for determination of paranitrophenol in water sample [16]. However, as far as we are aware, there are no reports about AuNCs coupled 
with SMIPs. Hence, in this study, we attempt to propose SMIPcoated AuNCs based fluorescent sensor by selecting bisphenol A (BPA) as model analyte.

BPA, one important phenolic environmental estrogens (PEEs), has shown adverse properties, namely, inducing estrogenic endocrine disruption and promoting tumor progression as an important intermediate in the industrial manufacture of several plastics $[17,18]$. For that reason, the identification and determination of trace amounts of BPA is very necessary. The most frequently used methods include high performance liquid chromatography (HPLC) [19], high performance liquid chromatography-mass spectrometry (MS) [20], and gas chromatography-mass spectrometry (GC-MS) [21]. However, these methods are still involved in some problems such as complex instruments, high cost, time-consuming, and particularly low selectivity. So, MIPs with high selectivity are gaining popularity. Especially, MIPs based fluorescent detection for BPA has received increasing concerns [22-24]. For example, Kim et al. prepared a molecularly imprinted fluorescent sensor by using a CdSe QD as a signal transducer for BPA analysis [23]. In the meantime, Yuma et al. prepared magnetic MIPs by a multi-step swelling polymerization method for BPA followed by HPLC-fluorometry detection [24]. As seen, the MIPs coupled with fluorometry strategies are more convenient with their excellent merits.

Inspired by the above mentioned studies, we aspire to construct a new MIPs based AuNCs fluorescent sensor via a sol-gel polymerization process for selective recognition and sensitive detection of BPA on the basis of electron-transfer-induced fluorescence quenching. By making use of the powerful amido bonds between carboxyl-terminated AuNCs and amino-functionalized $\mathrm{SiO}_{2}$ nanoparticles, $\mathrm{SiO}_{2} @$ AuNCs were initially prepared; by using 3-aminopropyltriethoxy silane as functional monomer and tetraethoxysilane as cross-linker, MIPs-coated AuNCs were then formed by anchoring MIP layer on the surface of $\mathrm{SiO}_{2} @ A u N C s$. The obtained $\mathrm{SiO}_{2} @$ AuNCs-MIPs were well characterized and their recognition/sensing properties were investigated in detail. The developed sensor was also successfully applied for the detection of BPA in seawater with satisfactory results.

\section{Experimental}

\subsection{Reagents}

Hydrogen tetrachloroaurate trihydrate $\left(\mathrm{HAuCl}_{4} \cdot 3 \mathrm{H}_{2} \mathrm{O}\right)$ was provided by Alfa Aesar (Tianjin, China). L-Glutathione in the reduced form (GSH), 1-(3-dimethylaminopropyl)-3-ethylcarbo diimide hydrochloride (EDC), 1-hydroxy-5-pyrrolidinedione (NHS) and 2-morpholino-ethanesulfonic acid (MES) were obtained from Aladdin (Shanghai, China). 3-Aminopropyltriethoxysilane (APTES), tetramethoxysilane (TEOS) and phosphate-buffered saline (PBS) were obtained from J\&K Chemical Ltd. (Beijing, China). BPA, hydroquinone, estradiol, phenol, and cholesterol were purchased from Sigma-Aldrich (Shanghai, China). Other reagents and materials were all supplied by Sinopharm Chemical Reagent (Shanghai, China). Aqueous solutions were prepared with ultrapure water (18.2 M $\Omega$ specific resistance) obtained with a Pall Cascada laboratory water system (Millipore, Bedford, MA, USA).

\subsection{Instrumentation}

Fluorescence measurements were performed with a Fluoromax4 Spectrofluorometer (Horiba Scientific, Japan) equipped with $1 \mathrm{~cm}$ quartz cell at $25^{\circ} \mathrm{C}$, with excitation and emission slit widths of 5 and $5 \mathrm{~nm}$, respectively, and the excitation wavelength at $396 \mathrm{~nm}$. The morphological evaluation was examined with a scanning electron microscope (SEM, Hitachi S-4800 FE-SEM, operating at $5 \mathrm{kV}$ ) and a transmission electron microscope (TEM, JEM-2100F). The HPLC-UV instrumental (Skyray Instrument Inc., China) conditions employed for BPA determination were as follows: analytical column, a $\mathrm{C}_{18}$ column with $250 \mathrm{~mm} \times 4.6 \mathrm{~mm}$ i.d. (Arcus EP- $\mathrm{C}_{18}, 5 \mu \mathrm{m}$, Waters, USA); mobile phase, acetonitrile/water (80:20, v/v); flow rate, $1.0 \mathrm{~mL} \mathrm{~min}^{-1}$; detection wavelength, $228 \mathrm{~nm}$; room temperature.

\subsection{Synthesis of carboxyl-terminated AuNCS}

The carboxyl-terminated AuNCs were synthesized according to the process described in previous study [25]. Briefly, freshly prepared aqueous solutions of $\mathrm{HAuCl}_{4}(20 \mathrm{mM}, 0.50 \mathrm{~mL})$ and $\mathrm{GSH}$ $(100 \mathrm{mM}, 0.15 \mathrm{~mL})$ were mixed with $4.35 \mathrm{~mL}$ of ultrapure water at $25^{\circ} \mathrm{C}$. The reaction mixture was heated to $70^{\circ} \mathrm{C}$ under gentle stirring ( $500 \mathrm{rpm}$ ) for $24 \mathrm{~h}$. As a result, a strong orange AuNCs aqueous solution under $365 \mathrm{~nm}$ UV-light was formed, and it could be stored at $4{ }^{\circ} \mathrm{C}$ for 6 months with negligible changes in their optical properties.

\subsection{Synthesis of amino-functionalized $\mathrm{SiO}_{2}$}

Uniform amino-functionalized $\mathrm{SiO}_{2}$ nanoparticles were synthesized by hydrolysis of TEOS with aqueous ammonia $\left(\mathrm{NH}_{3} \cdot \mathrm{H}_{2} \mathrm{O}\right)$, followed by APTES functionalization, according to the reported methods [26-28] with slight modification. In a word, ethanol $(30 \mathrm{~mL})$ and ultrapure water $(50 \mathrm{~mL})$ were added to a $250 \mathrm{~mL}$ threenecked, round-bottom flask. Under vigorous stirring, $\mathrm{NH}_{3} \cdot \mathrm{H}_{2} \mathrm{O}$ $(10 \mathrm{~mL})$ was added to the above solution, followed by adding the mixture of ethanol $(20 \mathrm{~mL})$ and TEOS $(5 \mathrm{~mL})$ drop by drop, and then was kept stirring overnight. APTES ( $5 \mathrm{~mL}$ ) was then added, being stirred for additional $12 \mathrm{~h}$. The resultant mixture was separated by centrifuge and washed with ethanol at least five times. Finally, the modified nanoparticles were dried under vacuum at $40^{\circ} \mathrm{C}$ for $12 \mathrm{~h}$.

\subsection{Preparation of $\mathrm{SiO}_{2} @ A u N C s$}

$\mathrm{SiO}_{2} @ A u N C s$ were prepared according to the reported procedure $[29,30]$ with necessary modification. Typically, EDC $(20 \mathrm{mg} / \mathrm{mL}$ in MES buffer $(\mathrm{pH}=5.2,0.1 \mathrm{mM}), 6 \mathrm{~mL})$ was mixed with carboxyl-terminated AuNCs aqueous solution $(10 \mathrm{~mL})$ for 10 min until the solution color became slightly turbid yellow from light yellow. Then, NHS $(10 \mathrm{mg} / \mathrm{mL}, 6 \mathrm{~mL})$ was added and mixed uniformly. Meanwhile, amino-functionalized $\mathrm{SiO}_{2}$ particles $(60 \mathrm{mg})$ were uniformly dispersed in MES buffer $(\mathrm{pH}=5.2$, $0.1 \mathrm{mM}, 50 \mathrm{~mL}$ ), followed by the addition of the above resultant AuNCs solution drop by drop. The mixture solution was stirred for $12 \mathrm{~h}$ at room temperature in the dark. Finally, the obtained $\mathrm{SiO}_{2} @$ AuNCs composite nanoparticles were centrifuged (unbound AuNCs were removed) and then washed with PBS (0.01 M, pH = 7.0).

\subsection{Preparation of $\mathrm{SiO}_{2} @ A u N C s-M I P s$}

Initially, the $\mathrm{SiO}_{2} @ A u N C s$ nanoparticles were dispersed in PBS $(0.01 \mathrm{M}, \mathrm{pH}=7.0,40 \mathrm{~mL})$. The pre-polymerized solution of functional monomer (APTES) and template (BPA) (sol-gel prepolymerization for $12 \mathrm{~h}$ ) was added, and was stirred for $30 \mathrm{~min}$. And then, $\mathrm{NH}_{3} \cdot \mathrm{H}_{2} \mathrm{O}(100 \mu \mathrm{L})$ and TEOS $(80 \mu \mathrm{L})$ were orderly added to the above solution, stirring for $12 \mathrm{~h}$. Finally, yellow nanoparticles were obtained after the removal of embedded template BPA by elution with a mixture of methanol and acetic acid (9:1) for $24 \mathrm{~h}$ until no BPA was detected by UV-vis. The products were indicated $\mathrm{SiO}_{2} @$ AuNCs-MIPs (MIPs for simplicity). As a control, the 
hybrid structured non-imprinted polymers, namely $\mathrm{SiO}_{2} @ A u N C s-$ NIPs (NIPs for simplicity) were prepared under the same conditions in the absence of the template BPA.

\subsection{Fluorescence measurement}

In the experiments, all the fluorescence intensity measurements were performed under the same conditions: the slit widths of the excitation and emission were both $5 \mathrm{~nm}$, the excitation wavelength was set at $396 \mathrm{~nm}$ with a recording emission range of $450-750 \mathrm{~nm}$, and the photomultiplier tube voltage was set at 700 V. $\mathrm{SiO}_{2} @ A u N C s-M I P s$ or $\mathrm{SiO}_{2} @ A u N C s-N I P s$ were added into BPA (or its analogs) solutions. The mixture was mixed thoroughly and scanned by the Fluoromax-4 Spectrofluorometer. Between the different determinations, the $\mathrm{SiO}_{2} @$ AuNCs-MIPs were stored at $4{ }^{\circ} \mathrm{C}$ in dark.

\subsection{Analysis of seawater samples}

Surface seawater samples were collected into a teflon bottle from the Fisherman's Wharf of the Yellow Sea located in the coastal zone area of Yantai City. $10 \mathrm{~mL}$ seawater samples were filtered through a $0.45 \mu \mathrm{m}$ microporous membrane filter, 1,2 and $5 \mu \mathrm{M} \mathrm{BPA}$ standard solutions were added into the treated seawater samples for spiked test. Prior to analysis, $10 \mathrm{~mL}$ of the acetonitrile was used to extract and transfer BPA to glass test tubes, and then acetonitrile was evaporated by a brief treatment with a stream of nitrogen. Then, $2 \mathrm{~mL}$ PBS solution was added for re-dissolution, followed by the subsequent $\mathrm{SiO}_{2} @$ AuNCs-MIPs treatment and fluorescence measurement.

\section{Results and discussion}

\subsection{Preparation and characterization of $\mathrm{SiO}_{2} @ A u N C s-M I P s$}

Fig. 1 schematically shows the preparation and sensing process of $\mathrm{SiO}_{2} @$ AuNCs-MIPs. Monodispersed $\mathrm{SiO}_{2}$ nanoparticles were synthesized by the Stöber method $[26,27]$ and subsequently formed the amino-functionalized $\mathrm{SiO}_{2}$ modified with APTES [28]. AuNCs were prepared using GSH as stabilizer and reducing reagent in water phase in a more simple way. Fig. S1 shows its photoemission at $396 \mathrm{~nm}$ excitation wavelength and the inset demonstrates orange color solution under $365 \mathrm{~nm}$ UV-light. The carboxylterminated AuNCs could conjugate with amino-functionalized $\mathrm{SiO}_{2}$ by generating powerful amido bonds. Then, the resultant $\mathrm{SiO}_{2} @ A u N C s$ composites coupled with APTES and TEOS to produce polymeric networks under $\mathrm{NH}_{3} \cdot \mathrm{H}_{2} \mathrm{O}$ catalyzing around BPA. That is, MIPs-coated AuNCs were formed by anchoring MIP layer on the surface of $\mathrm{SiO}_{2} @ A u N C s$ via a sol-gel process. After removing the template BPA, the $\mathrm{SiO}_{2} @ A u N C s-M I P s$ were obtained, resulting in strong fluorescence. However, when BPA was rebound, fluorescence quenching of AuNCs would occur. So, it is possible to sense BPA fluorescently.

In order to get effective and favorable MIPs, condition optimization experiments were carried out, such as the amounts of APTES and TEOS. As seen in Fig. S2, $60 \mu \mathrm{L}$ of APTES and $80 \mu \mathrm{L}$ of TEOS were used in the following tests. The amount of the AuNCs was also investigated, and in the end, $10 \mathrm{~mL}$ of AuNCs were selected in the following work.

To further confirm the successful preparation of the hybrid structured $\mathrm{SiO}_{2} @ A u N C s-M I P s$, photographs under visible light and 365 nm UV-light of the AuNCs, $\mathrm{SiO}_{2} @ A u N C s, \mathrm{SiO}_{2} @ A u N C s$ with adding BPA, and $\mathrm{SiO}_{2} @ A u N C s-M I P s$ were displayed. As seen in Fig. S3a, the colors were light yellow, yellow suspension, white suspension and light yellow suspension, respectively. Fig. S3b shows their corresponding color changes under $365 \mathrm{~nm}$ UV-light, orange, dark orange, light pink and dark orange, respectively. These results suggested that the $\mathrm{SiO}_{2} @ A u N C s-M I P s$ with fluorescence properties were prepared successfully.

SEM (Fig. 2A) and TEM images (Fig. 2B-D) were shown to characterize material morphology. As seen from Fig. $2 \mathrm{~A}$ and $\mathrm{B}$, the diameter of the uniform $\mathrm{SiO}_{2}$ was about $80 \mathrm{~nm}$. The AuNCs on its surface displaying highly spherical shape were $5-10 \mathrm{~nm}$ in diameter (Fig. 2C, inset), which played an important role of well-defined support substrates for nanostructured imprinted materials (Fig. 2C and D). In addition, it was observed from Fig. 2D that the thin MIP layer was about $5 \mathrm{~nm}$ in the outmost layer, which provided better site accessibility for special recognition toward the corresponding template [31].

FT-IR spectra were employed to investigate the synthesized nanomaterials. As seen in Fig. S4a, the characteristic signals at 1095 and $798 \mathrm{~cm}^{-1}$ could be attributed to the stretching vibration of $\mathrm{Si}-\mathrm{O}-\mathrm{Si}$ and $\mathrm{Si}-\mathrm{O}$, respectively. The peaks at 3413 and $1643 \mathrm{~cm}^{-1}$ (Fig. S4a) proved the amino group of APTES was successfully modified onto the surface of $\mathrm{SiO}_{2}$. The successful synthesis of $\mathrm{SiO}_{2} @$ AuNCs composite was further studied as shown in Fig. S4b, presenting peaks at 1411,1554 and $1635 \mathrm{~cm}^{-1}$ from the vibration of $\mathrm{CO}-\mathrm{NH}$. Because there is no introduction of new functional groups, the spectrum had no obvious change for the $\mathrm{SiO}_{2} @ A u N C s-M I P s$, as shown in Fig. S4c.

\subsection{Possible sensing mechanism of the $\mathrm{SiO}_{2} @ A u N C s-M I P s$}

The fluorescence quenching in this system followed the Stern-Volmer Equation [32-34] as follows,

$\frac{F_{0}}{F}=1+K_{\mathrm{sv}} C_{q}$

where $F_{0}$ and $F$ are the fluorescence intensities in the absence and presence of quencher, respectively, $C_{q}$ is the concentration of the quencher, and $K_{\mathrm{sv}}$ is the quenching constant for the quencher. The ratio of $K_{\mathrm{sv}, \mathrm{MIP}}$ to $K_{\mathrm{sv}, \mathrm{NIP}}$ was defined as the imprinting factor, and $\left(F_{0} / F\right)-1$ was defined as the quenching amount. In general, fluorescence quenching includes two quenching modes, namely, dynamic and static quenching $[32,33]$. In view of the available experimental conditions of our lab, the absorption spectra of $\mathrm{SiO}_{2} @$ AuNCsMIPs were tested to check the quenching mechanism [32,33]. The absorption spectra of $\mathrm{SiO}_{2} @$ AuNCs-MIPs changed with the addition of the quencher, hence it could be deduced the fluorescence quenching belonged to static quenching.

Meanwhile, the recognition process for BPA by the prepared $\mathrm{SiO}_{2} @$ AuNCs-MIPs was schematically shown in Fig. 1. As seen, the fluorescence quenching could be ascribed to the electron transfer between AuNCs and BPA. Moreover, the quenching mechanism could be more clearly illustrated in Fig. 3A. It has been reported that a strong charge-transfer interaction could occur between this electron-rich aromatic ring (conjugating - $\mathrm{OH}$ ) and electrondeficient amino group $[35,36]$. Herein, the electron transfer would result in the formation of a Meisenheimer complex between BPA and the primary amino groups on the surface of the AuNCs. Then the photo-induced energy of AuNCs would be transferred to the complex, leading to fluorescence quenching of AuNCs. As well as, the absorption peak and yellow color of AuNCs disappeared (Fig. 3B, curve $\mathrm{b}$ and inset $\mathrm{b}$ ). Therefore, BPA could be sensed fluorescently in this way.

\subsection{Analytical sensitivity of the sensor}

Based on the above results, the sensor could be employed for the quantitative determination of BPA. For this purpose, the concentrations of $\mathrm{SiO}_{2} @ A u N C s-M I P s$ solution were first examined. As shown in Fig. S5, the highest quenching amount appeared at 

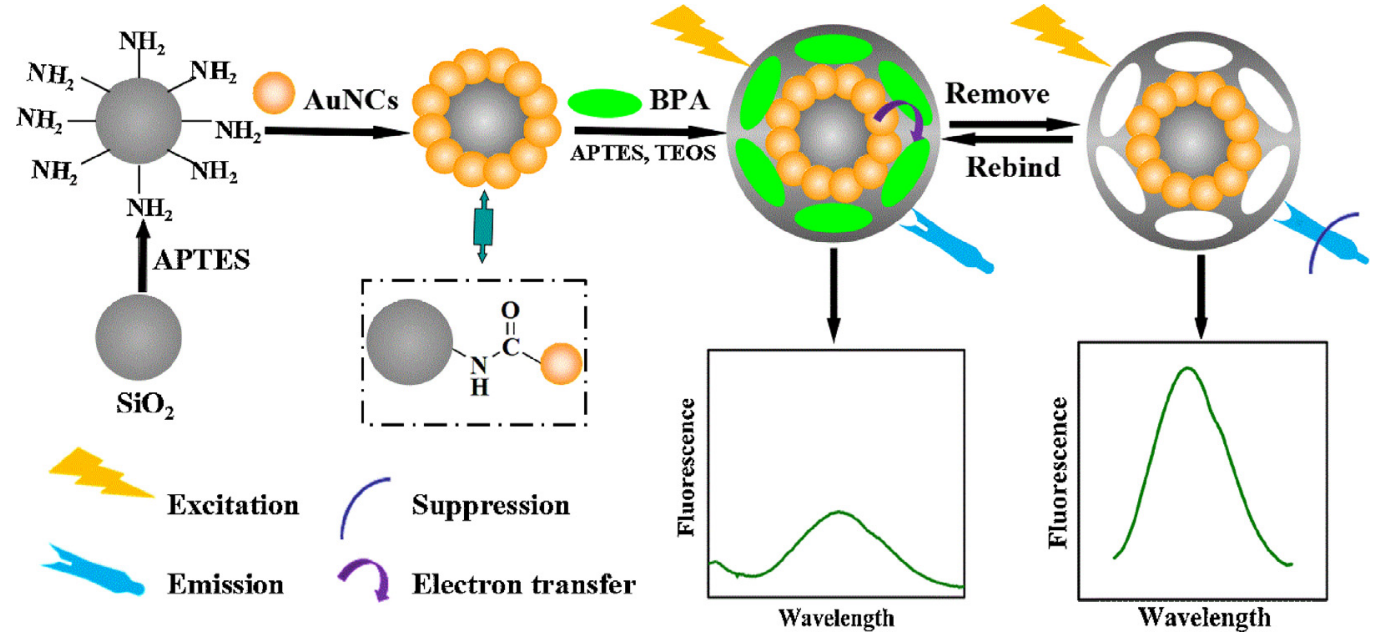

Fig. 1. Schematic illustration of the process for the preparation of the $\mathrm{SiO}_{2} @ A u N C s-M I P s$.

$25 \mathrm{mg} / \mathrm{L}$. So, $25 \mathrm{mg} / \mathrm{LSiO}_{2} @$ AuNCs-MIPs solution was chosen. Then, the response time was also tested, as well as the binding properties of the MIP sensor and its corresponding NIP. As seen from Fig. 4A, when the BPA concentration was fixed, the fluorescence intensity decreased rapidly with time increasing in the first $25 \mathrm{~min}$, and then the curve became flat. Hence, 25 min was selected as the response time for the following experiments.

As shown in Fig. 4B, the fluorescence intensities of the $\mathrm{SiO}_{2} @$ AuNCs-MIPs decreased linearly with the increase of BPA concentrations over the range of $0-13.1 \mu \mathrm{M}$, with a correlation coefficient of 0.9942 . And a favorable limit of detection (LOD, $\mathrm{S} / \mathrm{N}=3$ ) of $0.10 \mu \mathrm{M}$ was obtained, which meets with the requirement of trace analysis. Besides, this value is lower than the permitted maximum contaminant level of $0.05 \mathrm{mg} / \mathrm{L}(0.2 \mu \mathrm{M})$ for BPA in environmental water safety monitoring. On the contrast, the decrease of fluorescence intensity of corresponding NIPs was much lower at the same concentration of BPA, as seen in Fig. 4C. These results suggested that the specific molecular recognition sites with predetermined selectivity were formed in the MIPs, while the NIPs had no imprinting sites; the rebinding of BPA took place at
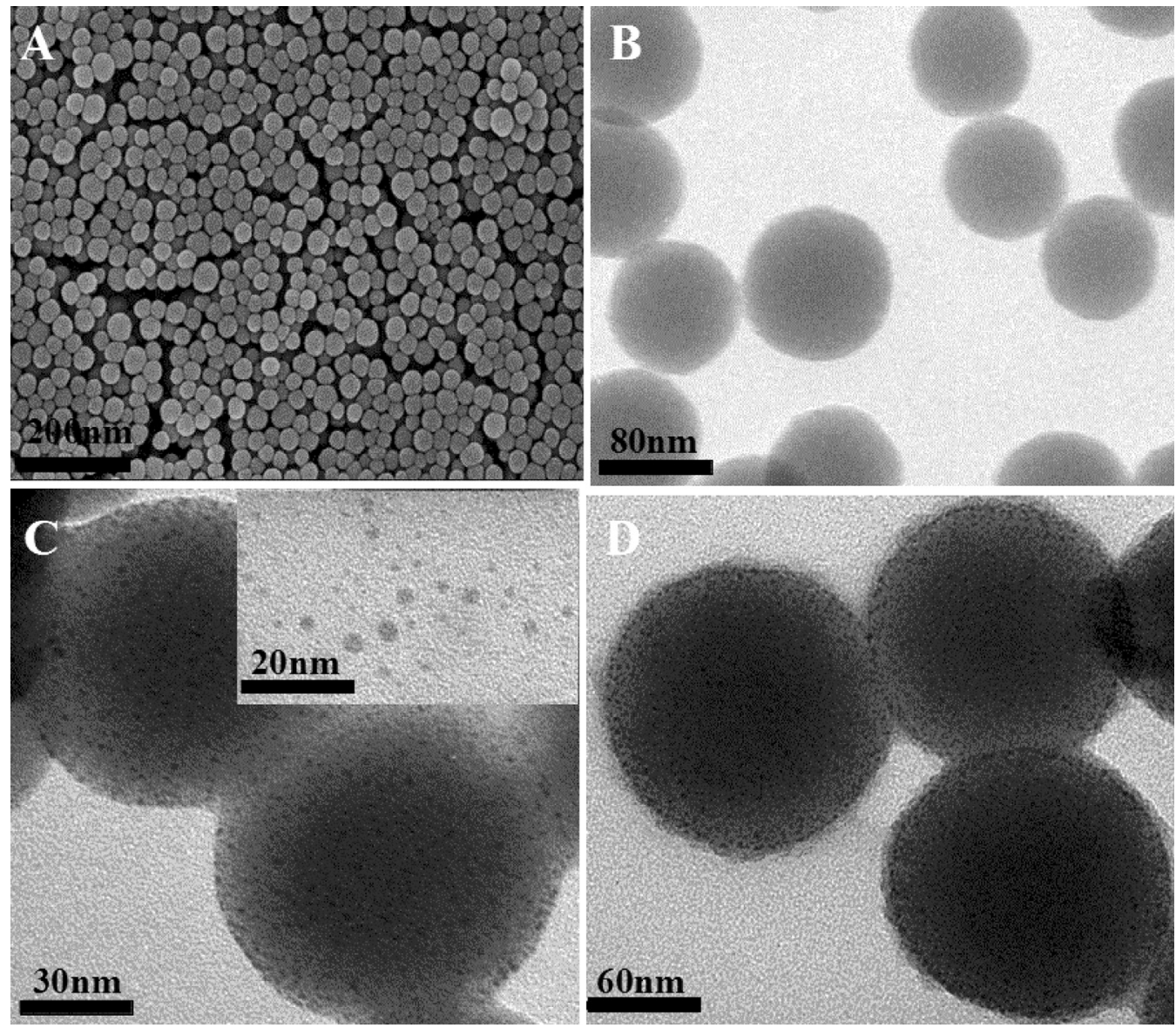

Fig. 2. (A) SEM image and (B) TEM image of $\mathrm{SiO}_{2}$, (C) TEM image of $\mathrm{SiO}_{2} @ A u N C s,(D)$ TEM image of $\mathrm{SiO}_{2} @ A u N C s-M I P s$ (inset C: TEM image of AuNCs). 

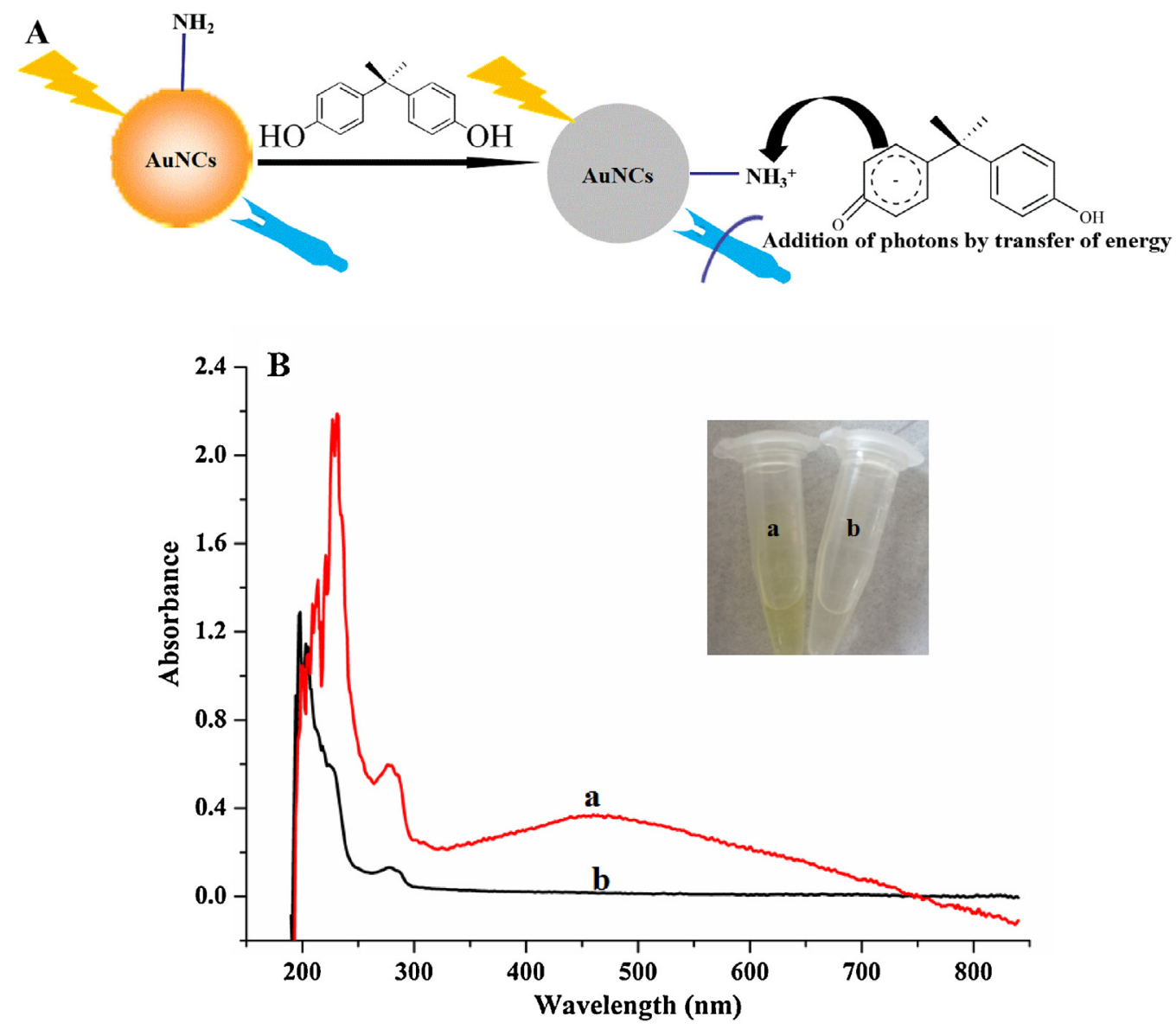

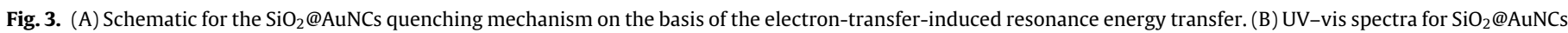
solution with adding APTES (a) and $\mathrm{SiO}_{2} @ A$ ANCs with adding the APTES and BPA (b), and the insets show their corresponding solution photographs.

the imprinted sites. According to Equation 1, the ratio of $K_{\mathrm{sv}, \mathrm{MIP}}$ to $K_{\mathrm{sv}, \mathrm{NIP}}$ could be defined as the imprinting factor, so, herein, the imprinting factor was attained of 2.7. In addition, for the MIP sensor, the precision for the five-replicate detections of BPA was 1.5\% (relative standard deviation, RSD). Therefore, the MIP based sensor could quickly, sensitively and accurately detect BPA.

\subsection{Fluorescent stability and repeatability of the sensor}

Fluorescence stability of the $\mathrm{SiO}_{2} @ A u N C s-M I P s$ sensor was evaluated by the repeated detection of the fluorescence emission intensity every $10 \mathrm{~min}$. The results shown in Fig. 5 indicated a stable emission of AuNCs within $60 \mathrm{~min}$. Moreover, the fluorescence intensity of the sensor during the storage was also investigated. The repeated detection of the fluorescence emission intensity was performed every day. As seen in Fig. S6, after the sensor was stored for 7 days, the intensity retained $93 \%$ of its initial response. This result implied that the developed fluorescence sensor has acceptable storage stability. The satisfying fluorescence stability might well be attributed to the excellent protection of MIP coatings.

As is well known, desorption and regeneration is an important index for the application of MIPs. BPA adsorption-desorption procedure was repeated four times by using the same $\mathrm{SiO}_{2} @ A u N C s-$ MIPs sensor. As shown in Fig. S7, the sensor could retain its fluorescence intensity and detection sensitivity, presenting relatively low standard errors within $1.8 \%$ during three recycles. The results indicated that sensor retained their recovery efficiency, which was a clear superiority over disposable materials and thereby it could be used repeatedly.

\subsection{Selectivity of the sensor}

In order to estimate the selectivity of the $\mathrm{SiO}_{2} @ A u N C s-M I P s$ sensor, several compounds, including hydrochinone, phenol, estradiol and cholesterol with molecular structures or molecular weights similar to BPA (Fig. S8), were tested, respectively. As seen from Fig. 6A, the MIP sensor displayed significant change of fluorescent intensity toward BPA, much larger than that of its analogs. The difference was very likely due to the difference between BPA and the analogs in molecular weight, spatial structure and interaction with APTES. As for hydrochinone, it has symmetrical structure similar to BPA, and can interact with the APTES, so it can easily access the recognition sites and partly quench the florescence. Phenol is smaller molecule, and it entered into the sites easily, as well as the specific recognition sites were partly complementary to phenol, so a certain fluorescence quenching occurred. For estradiol and cholesterol, it is difficult to attach to the binding sites because of their high molecular weights, and thereby led to very low quenching amounts. In addition, there was no significant difference of the fluorescence intensity between the five compounds for NIPs. Therefore, the MIP sensor had high selectivity toward BPA.

As well, the competitive binding experiments were performed by changing the ratio of different concentrations of hydroquinone and the fixed concentration of BPA. As shown in Fig. 6B, the fluorescence intensity of $\mathrm{SiO}_{2} @ A u N C s-M I P s$ sensor had no obvious change with the increase of $C_{\mathrm{HYD}} / C_{\mathrm{BPA}}$ ratio (hydroquinone was named $\mathrm{HYD}$ for convenience). Through the competitive experiments, it was further confirmed that the sensor had excellent specificity toward the template BPA. 

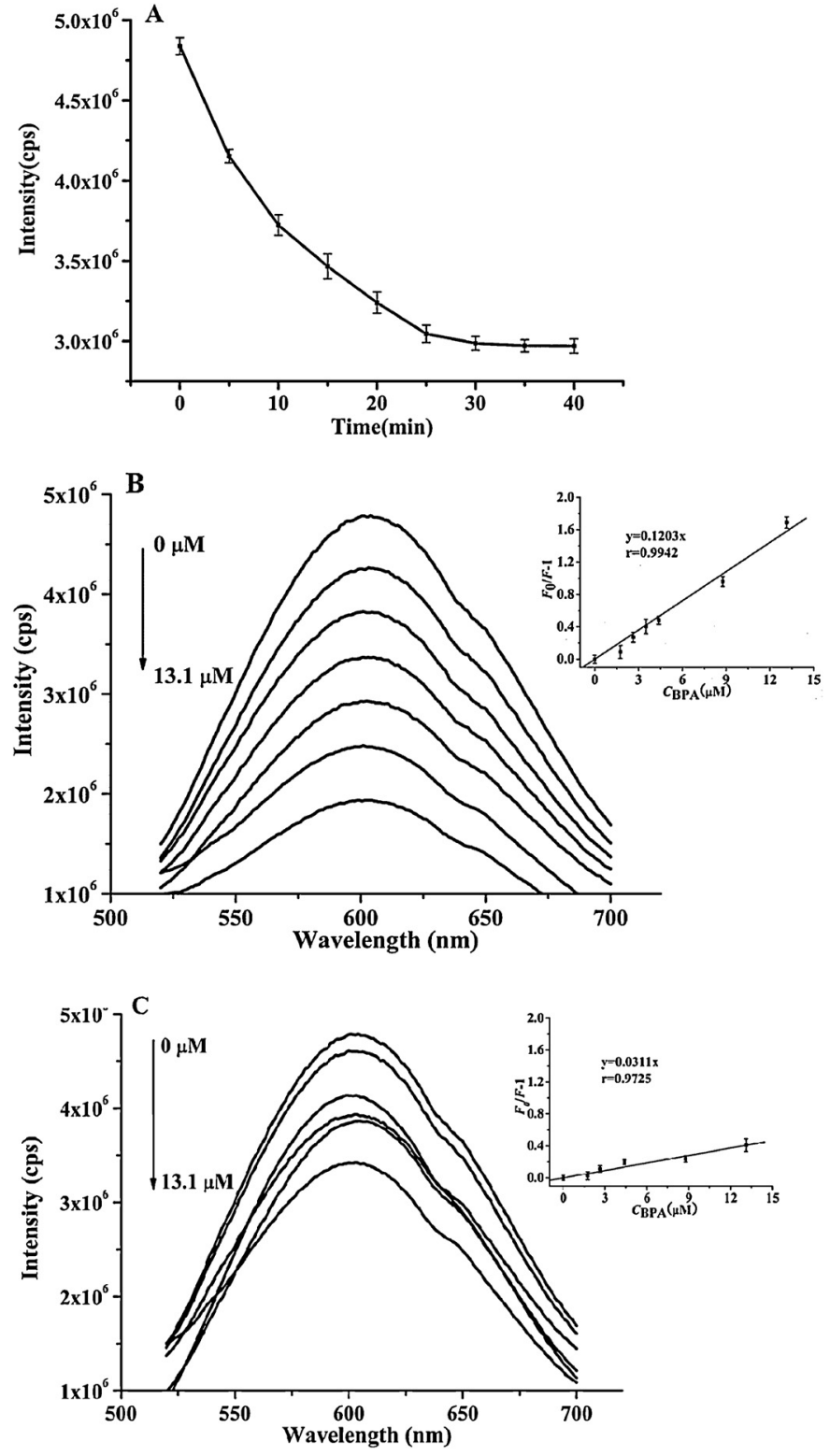

Fig. 4. (A) Fluorescence response time of $\mathrm{SiO}_{2} @ A u N C s-M I P s$ for BPA. (B) and (C): Fluorescence emission spectra of $\mathrm{SiO}_{2} @ A u N C s-M I P s$ and $\mathrm{SiO}_{2} @ A u N C s-N I P s$ with addition of the indicated concentrations of BPA, respectively. The inset graphs show fluorescence intensity corresponding Stern-Volmer plots. The experimental conditions were $\mathrm{SiO}_{2} @ A u N C s-M I P$ or $\mathrm{SiO}_{2} @ A u N C s-N I P 25 \mathrm{mg} / \mathrm{L} ; C_{\mathrm{BPA}}, 3.4 \mu \mathrm{M}$; excited light: $396 \mathrm{~nm}$.

Table 1

Spiked recoveries and relative standard deviations (RSD, \%, $n=5$ ) in seawater using $\mathrm{SiO}_{2} @$ AuNCs-MIPs sensor and HPLC-UV analysis.

\begin{tabular}{llllll}
\hline \multirow{2}{*}{ Spiked $(\mu \mathrm{M})$} & \multicolumn{2}{l}{$\mathrm{SiO}_{2} @$ AuNCs-MIPs sensor } & & \multicolumn{2}{l}{ HPLC-UV } \\
\cline { 2 - 3 } & Recovery (\%) & RSD (\%) & & Recovery (\%) & RSD (\%) \\
\hline 1.00 & 92.8 & 3.4 & & 97.6 & 2.3 \\
2.00 & 96.2 & 3.1 & & 103 & 1.8 \\
5.00 & 91.3 & 4.8 & & 102 & 1.4 \\
\hline
\end{tabular}

\subsection{Practical application}

In order to further investigate the practical applicability of the developed $\mathrm{SiO}_{2} @$ AuNCs-MIPs sensor, seawater samples were selected as the real complicated samples. As listed in Table 1, satisfactory recoveries were obtained in a range of $91.3-96.2 \%$ with RSD

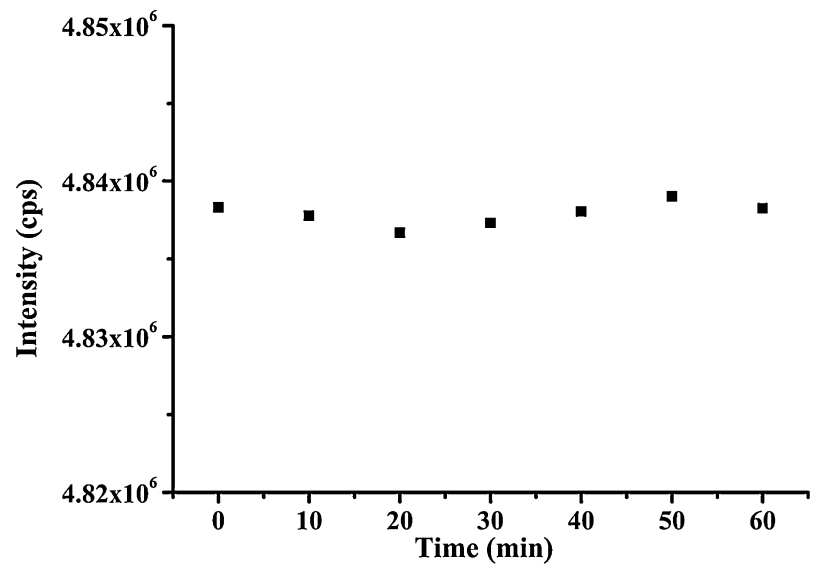

Fig. 5. Fluorescence intensity of $25 \mathrm{mg} / \mathrm{L} \mathrm{SiO}_{2} @ A u N C s-M I P s$ solution within $60 \mathrm{~min}$.
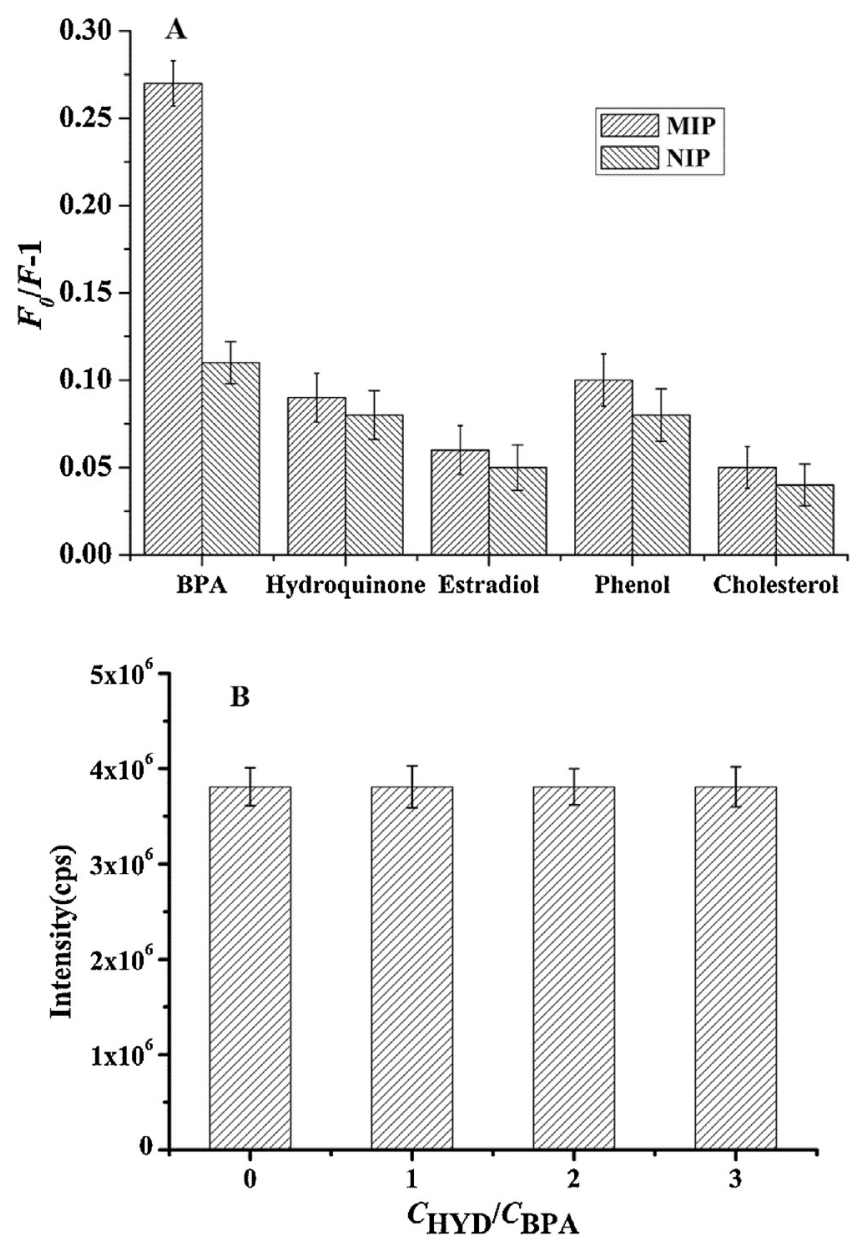

Fig. 6. (A) Binding behaviors of different analogs at the same initial concentration on the $\mathrm{SiO}_{2} @ A u N C s-M I P s$ and $\mathrm{SiO}_{2} @ A u N C s-N I P s$. (B) Effects of hydroquinone on the binding capacity under the different concentration of hydroquinone and the fixed concentration of BPA on the $\mathrm{SiO}_{2} @ A u N C s-M I P s$. Experimental conditions: $\mathrm{SiO}_{2} @ A u N C s-M I P s$ or $\mathrm{SiO}_{2} @ A u N C s-N I P s, 25 \mathrm{mg} / \mathrm{L} ; C_{\mathrm{BPA}}, 2.6 \mu \mathrm{M}$; excited light: $396 \mathrm{~nm}$.

of 3.1-4.8\% for the BPA at three spiked concentrations. The values were comparable to that obtained by HPLC-UV, namely $97.6-103 \%$ with RSD of $1.4-2.3 \%$ (Table 1 ). These results clearly confirmed that the developed $\mathrm{SiO}_{2} @$ AuNCs-MIPs sensor was potentially applicable for the accurate determination of BPA in real samples. 


\subsection{Method performance comparison}

The performance of the developed fluorescence method for the determination of BPA was compared with some reported approaches such as HPLC, electrochemistry, enzyme [37] and other coupling techniques. As listed from Table S1, methods like HPLC [19,38], GC-MS [21], LC/MS [20] and LC-MS/MS [39] can detect BPA with high sensitivity and selectivity, but they require complicated instruments and highly trained operators. The electrochemistry methods $[40,41]$ show low detection limit and broad linear range, but possibly involve the disadvantages of instability and interference of organics in anodic/catholic stripping voltammetry. Besides, magnetic MIPs for BPA followed by HPLC-fluorometry determination involve complicated sample treatment [24]. A molecularly imprinted fluorescent sensor using CdSe QD as signal transducer for BPA detection needs time-consuming synthesizing process [23]. However, our method based on the combination of MIPs and AuNCs only needs a simple step, and it can be successfully applied for the detection of BPA in complicated matrices such as seawater samples. On the other hand, we also carried out HPLC-UV experiments for BPA determination. Compared to the LOD of $0.87 \mu \mathrm{M}$, the sensor method with LOD of $0.1 \mu \mathrm{M}$ is more sensitive. In terms of detection time, the HPLC method requires more than 6 min. However, the sensor is timesaving within $1 \mathrm{~min}$ to complete the detection. As a result, the present sensor method in our study has remarkable advantages such as simplicity, rapidity and universality, high selectivity and sensitivity, and good reliability and practicability.

\section{Conclusions}

In summary, a novel $\mathrm{SiO}_{2} @ A u N C s-M I P s$ sensor was developed by sol-gel process for the detection of BPA on the basis of an electron-transfer-induced fluorescence quenching mechanism. By taking advantages of the high selectivity of MIPs and the strong fluorescence property of AuNCs, the sensor demonstrated a highly selective and sensitive recognition and determination of BPA. The simple, rapid, reliable and cost-effective sensing strategy provided an excellent fluorescent analysis platform for BPA monitoring. With the fast development of versatile MIPs and the gradual concern for AuNCs, we believe their synergistic effect can open up new opportunities to develop such composite materials for potential utilization. Besides, more explorations still need to be made to further improve the sensitivity of AuNCs-MIPs-based systems while retaining their high selectivity. Our effort along this line is currently underway.

\section{Acknowledgements}

This work was financially supported by the National Key Technology Research and Development Program of the Ministry of Science and Technology of China (2013BAD19B02), the National Key Scientific Instrument and Equipment Development Project (2013YQ17052506), the National Natural Science Foundation of China (21275158 and 21477160), and the Scientific Research Foundation for the Returned Overseas Chinese Scholars, State Education Ministry.

\section{Appendix A. Supplementary data}

Supplementary data associated with this article can be found, in the online version, at http://dx.doi.org/10.1016/j.snb.2015.01.115.

\section{References}

[1] J. Zheng, P.R. Nicovich, R.M. Dickson, Highly fluorescent noble-metal quantum dots, Annu. Rev. Phys. Chem. 58 (2007) 409-431.
[2] W. Zhang, X.W. He, Y. Chen, W.Y. Li, Y.K. Zhang, Molecularly imprinted polymer anchored on the surface of denatured bovine serum albumin modified CdTe quantum dots as fluorescent artificial receptor for recognition of target protein, Biosens. Bioelectron. 31 (2012) 84-89.

[3] D.Y. Li, X.W. He, Y. Chen, W.Y. Li, Y.K. Zhang, Novel hybrid structure silica/CdTe/molecularly imprinted polymer: synthesis, specific recognition, and quantitative fluorescence detection of bovine hemoglobin, ACS Appl. Mater. Interfaces 5 (2013) 12609-12616.

[4] H.B. Li, Y.L. Li, J. Cheng, Molecularly imprinted silica nanospheres embedded CdSe quantum dots for highly selective and sensitive optosensing of pyrethroids, Chem. Mater. 22 (2010) 2451-2457.

[5] T.T. Chen, Y.H. Hu, Y. Cen, X. Chu, Y. Lu, A dual-emission fluorescent nanocomplex of gold-cluster-decorated silica particles for live cell imaging of highly reactive oxygen species, J. Am. Chem. Soc. 135 (2013) 11595-11602.

[6] L. Shang, S.J. Dong, G.U. Nienhaus, Ultra-small fluorescent metal nanoclusters: synthesis and biological applications, Nano Today 6 (2011) 401-418.

[7] J.P. Xie, Y.G. Zheng, J.Y. Ying, Highly selective and ultrasensitive detection of $\mathrm{Hg}^{2+}$ based on fluorescence quenching of Au nanoclusters by $\mathrm{Hg}^{2+}-\mathrm{Au}^{+}$interactions, Chem. Commun. 46 (2010) 961-963.

[8] L.H. Jin, L. Shang, S.J. Guo, Y.X. Fang, D. Wen, L. Wang, J.Y. Yin, S.J. Dong, Biomolecule-stabilized Au nanoclusters as a fluorescence probe for sensitive detection of glucose, Biosens. Bioelectron. 26 (2011) 1965-1969.

[9] J.H. Li, Z. Zhang, S.F. Xu, L.X. Chen, N. Zhou, H. Xiong, H.L. Peng, Label-free colorimetric detection of trace cholesterol based on molecularly imprinted photonic hydrogels, J. Mater. Chem. 21 (2011) 19267-19274.

[10] L.X. Chen, S.F. Xu, J.H. Li, Recent advances in molecular imprinting technology: current status, challenges and highlighted applications, Chem. Soc. Rev. 40 (2011) 2922-2942.

[11] S. Suriyanarayanan, P.J. Cywinski, A.J. Moro, G.J. Mohr, W. Kutner, Chemosensors based on molecularly imprinted polymers, Top. Curr. Chem. 325 (2012) $165-265$.

[12] D.M. Gao, Z.P. Zhang, M.H. Wu, C.G. Xie, G.J. Guan, D.P. Wang, A surface functional monomer-directing strategy for highly dense imprinting of TNT at surface of silica nanoparticles, J. Am. Chem. Soc. 129 (2007) 7859-7866.

[13] M. Aliofkhazraei, N. Ali, Recent developments in miniaturization of sensor technologies and their applications, Comp. Mater. Process. 3 (2014) 245-306

[14] J.X. Liu, H. Chen, Z. Lin, J.M. Lin, Preparation of surface imprinting polymer capped Mn-doped ZnS quantum dots and their application for chemiluminescence detection of 4-nitrophenol in tap water, Anal. Chem. 82 (2010) 7380-7386.

[15] Y. Mao, Y. Bao, D.X. Han, F.H. Li, L. Niu, Efficient one-pot synthesis of molecularly imprinted silica nanospheres embedded carbon dots for fluorescent dopamine optosensing, Biosens. Bioelectron. 38 (2012) 55-60.

[16] Y. Zhou, Z.B. Qu, Y.B. Zeng, T.S. Zhou, G.Y. Shi, A novel composite of grapheme quantum dots and molecularly imprinted polymer for fluorescent detection of paranitrophenol, Biosens. Bioelectron. 52 (2014) 317-323.

[17] R.C. Dong, J.H. Li, H. Xiong, W.H. Lu, H.L. Peng, L.X. Chen, Thermosensitive molecularly imprinted polymers on porous carriers: preparation, characterization and properties as novel adsorbents for bisphenol A, Talanta 130 (2014) $182-191$

[18] V. Migeot, A. Dupuis, A. Cariot, M.A. Liaty, F. Pierre, S. Rabouan, Bisphenol A and its chlorinated derivatives in human colostrum, Environ. Sci. Technol. 47 (2013) 13791-13797.

[19] Y. Watabe, T. Kondo, H. Imai, M. Morita, N. Tanaka, K. Hosoya, Reducing bisphenol A contamination from analytical procedures to determine ultralow levels in environmental samples using automated HPLC microanalysis, Anal. Chem. 76 (2004) 105-109.

[20] S.M. Zimmers, E.P. Brownea, P.W. Okeefe, D.L. Anderton, L. Kramer, D.A. Reckhow, K.F. Arcaro, Determination of free bisphenol A (BPA) concentrations in breast milk of U.S. women using a sensitive LC/MS/MS method, Chemosphere 104 (2014) 237-243

[21] S. Gang, G. Yu, Z.X. Cai, Z.L. Zhang, Development of an analytical method to determine phenolic endocrine disrupting chemicals in sewage and sludge by GC/MS, Chin. Sci. Bull. 50 (2005) 2681-2687.

[22] Y.T. Wu, Y.J. Liu, X. Gao, K.C. Gao, H. Xia, M.F. Luo, X.J. Wang, L. Ye, Y. Shi, B. Lu, Monitoring bisphenol A and its biodegradation in water using a fluorescent molecularly imprinted chemosensor, Chemosphere 119 (2005) 515-523.

[23] Y.D. Kim, J.B. Jeon, J.Y. Chang, CdSe quantum dot-encapsulated molecularly imprinted mesoporous silica particles for fluorescent sensing of bisphenol A, J. Mater. Chem. 22 (2014) 24075-24080.

[24] Y. Hiratsuka, N. Funaya, H. Matsunaga, J. Haginaka, Preparation of magnetic molecularly imprinted polymers for bisphenol A and its analogues and their application to the assay of bisphenol A in river water, J. Pharm. Biomed. 75 (2013) 180-185

[25] Z.T. Luo, X. Yuan, Y. Yu, Q.B. Zhang, D.T. Leong, J.Y. Lee, J.P. Xie, From aggregation-induced emission of $\mathrm{Au}(\mathrm{I})$-thiolate complexes to ultrabright Au(0)@Au(I)-thiolate core-shell nanoclusters, J. Am. Chem. Soc. 134 (2012) $16662-16670$

[26] W. Stober, A. Fink, Controlled growth of monodisperse silica spheres in the micron size range, J. Colloid Interface Sci. 26 (1968) 62-69.

[27] R.X. Gao, JJ. Zhang X.W. He, L.X. Chen, Y.K. Zhang, Selective extraction of sulfonamides from food by use of silica-coated molecularly imprinted polymer nanospheres, Anal. Bioanal. Chem. 398 (2010) 451-461.

[28] L. Lu, R. Capek, A. Kornowski, N. Gaponik, A. Eychmuller, Selective fabrication of ordered bimetallic nanostructures with hierarchical porosity, Angew. Chem. Int. Ed. 44 (2005) 5997-6001. 
[29] K. Zhang, H.B. Zhou, O.S. Mei, S.H. Wang G.J. Guan, R.Y. Liu, J. Zhang, Z.P. Zhang, Instant visual detection of trinitrotoluene particulates on various surfaces by ratiometric fluorescence of dual-emission quantum dots hybrid, J. Am. Chem. Soc. 133 (2011) 8424-8427.

[30] L.Y. Chen, C.L. Chen, R.N. Li, Y. Li, S.Q. Liu, CdTe quantum dot functionalized silica nanosphere labels for ultrasensitive detection of biomarker, Chem. Commun. (2009) 2670-2672.

[31] Z. Lin, Z.W. Xia, J.N. Zheng, D. Zheng, L. Zhang, H.H. Yang, G.N. Chen, Synthesis of uniformly sized molecularly imprinted polymer-coated silica nanoparticles for selective recognition and enrichment of lysozyme, J. Mater. Chem. 22 (2012) 17914-17922.

[32] B.P. Kamat, J. Seetharamappa, Mechanism of interaction of vincristine sulphate and rifampicin with bovine serum albumin: a spectroscopic study, J. Chem. Sci. 117 (2005) 649-655.

[33] P.P.H. Cheng, D. Silvester, G.L. Wang, G. Kalyuzhny, A. Douglas, R.W. Murray, Dynamic and static quenching of fluorescence by $1-4 \mathrm{~nm}$ diameter gold monolayer-protected clusters, J. Phys. Chem. B 110 (2006) 4637-4644.

[34] R. Gopal, J.K. Lee, J.H. Lee, Y.G. Kim, G.C. Oh, C.H. Seo, Y.Y. Park, Effect of repetitive lysine-tryptophan motifs on the eukaryotic membrane, Int. J. Mol. Sci. 14(2013) 2190-2202.

[35] J.P. Colpa, C. MacLean, E.L. Mackor, Ions of odd alternant systems: proton complexes of aromatic hydrocarbons, Tetrahedron 19 (1963) 65-88.

[36] T. Gunnlaugsson, P.E. Kruger, T.C. Lee, R. Parkesh, F.M. Pfeffer, G.M. Hussey, Dual responsive chemosensors for anions: the combination of fluorescent PET (photoinduced electron transfer) and colorimetric chemosensors in a single molecule, Tetrahedron Lett. 44 (2003) 6575-6578.

[37] D.G. Mita, A. Attanasio, F. Arduini, N. Diano, V. Grano, U. Bencivenga, S. Rossi, A. Amine, D. Moscone, Enzymatic determination of BPA by means of tyrosinase immobilized on different carbon carriers, Biosens. Bioelectron. 23 (2007) 60-65.

[38] Y.F. Zhuang, M. Zhou, J. Gu, X.M. Li, Spectrophotometric and high performance liquid chromatographic methods for sensitive determination of bisphenol A Spectrochim. Acta A 122 (2014) 153-157.

[39] E. Maiolini, E. Ferri, A.L. Pitasi, A. Montoya, M.D. Giovanni, E. Errani, S. Girotti, Bisphenol A determination in baby bottles by chemiluminescence enzyme-linked immunosorbent assay, lateral flow immunoassay and liquid chromatography tandem mass spectrometry, Analyst 139 (2014) 318-324.

[40] H.S. Yin, Y.L. Zhou, J. Xu, S.Y. Ai, L. Cui, L.S. Zhu, Amperometric biosensor based on tyrosinase immobilized onto multiwalled carbon nanotubes-cobalt phthalocyanine-silk fibroin film and its application to determine bisphenol A, Anal. Chim. Acta 659 (2010) 144-150.

[41] H.S. Yin, Y.L. Zhou, S.Y. Ai, O.P. Chen, X.B. Zhu, X.G. Liu, L.S. Zhu, Sensitivity and selectivity determination of BPA in real water samples using PAMAM dendrimer and CoTe quantum dots modified glassy carbon electrode, J. Hazard. Mater. 174 (2010) 236-243.

\section{Biographies}

Xiaqing Wu received her BS degree in applied chemistry from Yanching Institute of Technology, China, in 2012, and in the same year she joined Dalian University, China. She is studying for her master degree. Her current research interest is molecularly imprinted polymers based chemical sensors for environmental analysis.

Zhong Zhang received his master degree from Nan Chang University, China, in 2012. Now, he is a doctoral candidate in Yantai Institute of Coastal Zone Research Chinese Academy of Sciences. His current research interest focuses on the preparation of novel molecularly imprinted polymers (MIPs) and their applications in chemo/biosensors and chromatographic separation analysis.

Jinhua Li received her PhD in analytical chemistry from the Department of Chemistry of Hong Kong Baptist University, Hong Kong SAR, China, in 2009. In the same year, she joined the Laboratory of Environmental Microanalysis and Monitoring in Yantai Institute of Coastal Zone Research, Chinese Academy of Sciences, as an assistant professor. Her current research interest focuses on preparation of novel molecular imprinting polymers and applications to sample pretreatment and chemo/biosensors.

Huiyan You received her PhD in analytical chemistry from Dalian Institute of Chemical Physics, Chinese Academy of Sciences, China. She engaged in the elec tric separation theory and application research for many years, participated in 10 scientific research projects and published more than 40 papers, 2 monographs. Now she is a professor in Dalian University. Her research interest is mainly centered on separation field using electrophoretic technique.

Yanbin Li obtained his $\mathrm{PhD}$ degree in agricultural engineering from Penn State University in 1989. He is a professor of biological engineering at the University of Arkansas, USA and Zhejiang University, China. His research focuses on biosensors for detection of foodborne pathogens and chemical residues and microbial prediction, modeling and simulation.

Lingxin Chen received his $\mathrm{PhD}$ in analytical chemistry from Dalian Institute of Chemical Physics, Chinese Academy of Sciences, China, in 2003. After two years postdoctoral experience in the Department of Chemistry, Tsinghua University, China, he joined first as a BK21 researcher and then as a research professor in the Department of Applied Chemistry, Hanyang University, Ansan, Korea, in 2006. Now he is a professor in Yantai Institute of Coastal Zone Research. Chinese Academy of Sciences. His current research interests include chromatographic separation techniques and optical sensor technologies for environmental analysis using novel properties of materials such as functionalized nanoparticles and molecularly imprinted polymers. He has published 2 monographs and more than 160 SCI peer-review journal papers. 